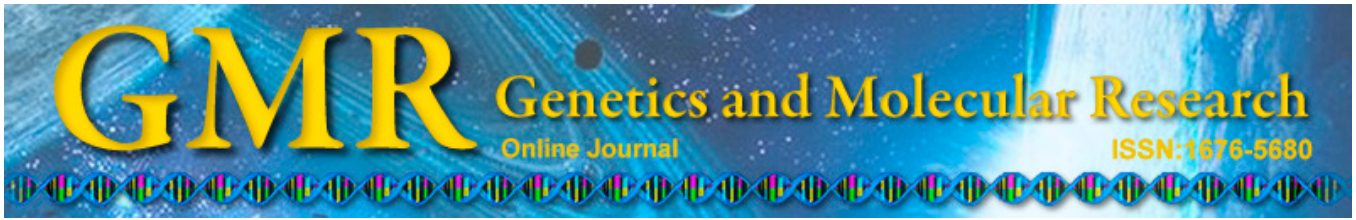

\title{
Effect of Tanshinone IIA intrathecal injections on pain and spinal inflammation in mice with bone tumors
}

\author{
B.X. Ren, Y. Ji, J.C. Tang, D.P. Sun, X. Hui, D.Q. Yang and X.L. Zhu \\ Anesthesiology Affiliated Hospital, University Jiangnan, Wuxi, \\ Jiangsu Province, China \\ Corresponding author: B.X. Ren \\ E-mail: renbingxu_rbx@163.com
}

Genet. Mol. Res. 14 (1): 2133-2138 (2015)

Received July 22, 2014

Accepted October 21, 2014

Published March 20, 2015

DOI http://dx.doi.org/10.4238/2015.March.20.24

\begin{abstract}
The study aimed to investigate the effect of intrathecal injections of Tanshinone IIA on thermal hyperalgesia in a mouse model of bone cancer-pain. Spinal IL-1 $\beta$, IL-6, TNF- $\alpha$ expression levels were analyzed. $\mathrm{C} 3 \mathrm{H} / \mathrm{HeNCrlVr}$ male mice were assigned to groups that either received dose-dependent injections of Tanshinone IIA, or the DMSO + Sham, Tanshinone IIA + Sham, DMSO + Tumor, and Control groups. Paw withdrawal thermal latency (PWTL) was measured with a radiant heat stimulus and mRNA expression levels were determined using real-time PCR. Fourteen days post-injection, PWTL in the DMSO + Tumor group was lower than that in the controls $(\mathrm{P}<0.05)$. Twentyone days post-injection, compared with the Control group, there was no significant difference in PWTL and IL-1 $\beta$, IL-6, and TNF- $\alpha$ expression levels between the Tanshinone IIA + Sham and DMSO + Sham groups $(\mathrm{P}>0.05)$. PWTL in the DMSO + Tumor group was significantly lower than the Control group $(\mathrm{P}<0.05)$, while the expression levels of IL$1 \beta$, IL- 6 , and TNF- $\alpha$ were significantly higher than controls. Compared with the DMSO + Tumor group, PWTLs were higher in the Tanshinone
\end{abstract}


IIA - 20- $\mu$ g and 40- $\mu$ g groups, while expression levels of IL-1 $\beta$, IL-6, and TNF- $\alpha$ were significantly lower $(\mathrm{P}<0.05)$. These measures were not significantly different between the Tanshinone IIA $10 \mu \mathrm{g}$ and the DMSO + Tumor groups $(\mathrm{P}>0.05)$. In conclusion, Tanshinone IIA may inhibit the release of inflammatory cytokines, such as, IL-1 $\beta$, IL-6 $\alpha$, TNF- $\alpha$.

Key words: Bone cancer pain; Spinal cord; Tanshinone IIA; IL-1 $\beta$; IL-6; TNF- $\alpha$

\section{INTRODUCTION}

During the formation of bone cancer, a large amount of dorsal horn astrocytes in the mouse spinal cord are stimulated (Ren et al., 2011), which produces large amounts of inflammatory substances, such as IL- $1 \beta$, IL- 6 , and TNF- $\alpha$. These play an important role in the signal transduction of many types of pain, including bone cancer pain (Marchand et al., 2005; Lu et al., 2011).

Salvia miltiorrhizae is a type of traditional Chinese medicine known to eliminate blood stasis, soothe the nerves, aid in apocenosis, and relieve pain (Dictionary of Traditional Chinese Medicine, 1986). However, its effective ingredients and mechanism of action in analgesia are not known. Tanshinone IIA is an ingredient of S. miltiorrhizae, and easily enters the nervous system due to its lipid solubility (Lin et al., 2002). Other studies have shown that Tanshinone IIA has anti-inflammatory properties (Fan et al., 2009). This study aims to establish a model of bone-cancer pain to study the effect of intrathecal injections of Tanshinone IIA on the pain behavior of mice and the expression levels of IL-1 $\beta$, IL-6, and TNF- $\alpha$ in the spine. It also explores the role and possible mechanism of Tanshinone IIA in bone cancer pain.

\section{MATERIAL AND METHODS}

\section{Materials}

Male $\mathrm{C} 3 \mathrm{H} / \mathrm{HeNCrlVr}$ mice (Vital River, China) were housed in a temperature-controlled room with a 12-h light/dark cycle and given food and water ad libitum. NCTC 2472 cell strain (ATCC, USA) was maintained in NCTC 135 media (Sigma, USA) containing 10\% horse sera (Gibco, USA). Tanshinone IIA was purchased from Nanjing Tcm Institute Of Chinese Materia Medica (China). ME-410C type thermal radiometer (Biomedical Engineering Institute of Chinese Academy of Sciences, China) was used to test paw withdrawal thermal latency (PWTL). PCR was run on ABI PRISM 7500 (Applied Biosystems, USA) and the results were visualized by the Gel Imaging System (Applied Biosystems).

\section{Method}

\section{Experiments}

Male $\mathrm{C} 3 \mathrm{H} / \mathrm{HeNCrlVr}$ mice $(\mathrm{N}=84)$ were used. A cancer pain model was designed according to an earlier study by Ren et al. (2011). Two weeks after beginning the pain model, 
behavioral tests and X-ray examinations were conducted to confirm whether the models were successfully established, and mice that did not meet requirements of the model were eliminated. There were 8 groups, with 12 mice each. There were 3 Cancer Pain groups: 1) Tanshinone IIA $10 \mu \mathrm{g}$, 2) Tanshinone IIA $20 \mu \mathrm{g}$, and 3) Tanshinone IIA $40 \mu \mathrm{g}$. After 14 days, these groups received intrathecal injections of $10-\mu \mathrm{g}, 20-\mu \mathrm{g}$, and $40-\mu \mathrm{g}$ Tanshinone IIA once a day for 7 days. In the 4) Control group, mice were allowed to breed normally for 21 days. 5) DMSO + Sham group: replace Tanshinone IIA with $20 \%$ DMSO $5 \mu \mathrm{L}$ to the Sham group. 6) Tanshinone IIA +Tumor group: replace $20 \%$ DMSO $5 \mu \mathrm{L}$ with Tanshinone IIA to the cancer pain groups. On the 21st day, the spinal cord lumbar enlargements of the mice in all groups were removed.

\section{Thermal hyperalgesia test}

PWTL was detected using a thermal radiation stimulator. Mice were placed in an organic glass box that had a $3-\mathrm{mm}$ thick glass pane bottom. The light from the thermal radiation stimulator could penetrate through the glass pane into the skin of the mouse foot. The irradiated limb presented a characteristic raise or retraction motion, and the time to raise or retract was recorded as the PWTL. The stimulator was removed after $20 \mathrm{~s}$ to prevent tissue damage. Each mouse was tested 5 times for $5 \mathrm{~min}$, and the average value was considered. The mice were then anesthetized and sacrificed. Their spinal cords were removed and immersed in liquid nitrogen for cryopreservation.

\section{Reverse transcription polymerase chain reaction (RT-PCR) test}

The primer was designed using the Primer Premier 5.0 software (Premier Biosoft, USA). Primer: $\beta$-actin: upstream 5'-CTGTCCCTGTATGCCTCTG-3', downstream 3'-GACAG GGACA TACGGA GAC-5'. IL-1 $\beta$ : upstream 5'-GCTTCAG GCAGGCAGTAT-3', downstream 5'-ACAAACCGCTTTT CCATCT-3'. IL-6: upstream 5'-TGGAGTCACAGAAGGAGT GGCTAAG-3', downstream 5'-TCTGACCACAGTGAGGAATGTCCAC-3'.TNF- $\alpha$ : upstream 5'-GTGGAACTGGCAG AAGAGG-3', downstream 5'-GGCTAC AGGCTTGTCACTC-3'. For total RNA extraction; 1) the spinal cord was cryopreserved and ground into liquid nitrogen, and the total tissue RNA was extracted with Trizol; 2) the OD 260 and 280 absorbencies were tested using the spectrophotometric method, and the total RNA concentration and purity of the sample was calculated; 3 ) reverse transcription to synthesize cDNA using a reverse transcription reagent kit was conducted; and 4) PCR was conducted using the $\mathrm{SYBR}^{\circledR}$ PrimeScript $^{\mathrm{TM}} \mathrm{RT}$ PCR reagent kit for the target genes GFAP, IL- $1 \beta$, IL-6, and TNF- $\alpha$. The $\beta$-actin gene was the internal reference, and an mRNA quantitative analysis was performed using the $2^{-\Delta \Delta c t}$ method; $\Delta \mathrm{ct}$ and $\Delta \Delta \mathrm{ct}$ were calculated according to the following formulae: $\Delta \mathrm{ct}=\mathrm{CT}$ value of the target gene - CT value of the reference gene; $\Delta \Delta \mathrm{ct}=\Delta \mathrm{ct}$ (treatment group) - $\Delta \mathrm{ct}$ (control group).

\section{Statistical analysis}

Statistical analyses were conducted using the SPSS 16.0 (SPSS Inc, USA) statistical software and a single factor variance components analysis. Results that met the threshold of $\mathrm{P}$ $<0.05$ were considered to be statistically significant. 


\section{RESULTS}

\section{PWTL comparison of all groups}

Before the intrathecal injections, there were no PWTL differences between groups (P $>0.05$ ). On the 14th day post-injection, PWTLs with the Sham injection were not statistically different $(\mathrm{P}>0.05)$ from those of the Control group, while the Cancer Pain group PWTLs dropped significantly $(\mathrm{P}<0.05)$. Three weeks after the injections, PWTLs of the Tanshinone $\mathrm{IIA}+$ Sham and DMSO + Sham groups were not significantly different $(\mathrm{P}>0.05)$ when compared with the Control group, while the Tanshinone IIA $20 \mu \mathrm{g}$ and Tanshinone II 40- $\mu$ g group PWTLs were higher than the DMSO + Tumor group, but lower than the Control group $(\mathrm{P}<$ 0.05). Tanshinone IIA 10- $\mu$ g group PWTLs were not significantly different from that of the Control group $(\mathrm{P}>0.05)$ (Table 1).

Table 1. Paw withdrawal thermal latency (PWTL) comparison of mice in all groups (S, means $\pm \mathrm{SD})$.

\begin{tabular}{lcccc}
\hline Groups & $\mathrm{N}$ & \multicolumn{3}{c}{ PWTL } \\
\cline { 3 - 5 } & & Before operation (0 days) & 14 days after the operation & 21 days after the operation \\
\hline Control group & 12 & $16.01 \pm 1.59$ & - & - \\
DMSO + Sham group & 12 & $15.97 \pm 1.49$ & $16.21 \pm 1.65$ & $16.31 \pm 2.94$ \\
DMSO + Tumor group & 12 & $16.38 \pm 1.87$ & $7.83 \pm 1.30^{\text {ab }}$ & $6.19 \pm 1.26^{\text {ab }}$ \\
Tanshinone IIA + Sham group & 12 & $16.80 \pm 1.79$ & $17.06 \pm 1.98$ & $17.01 \pm 2.43$ \\
Tanshinone IIA 10- $\mu$ g group & 12 & $16.64 \pm 1.81$ & $7.79 \pm 1.37^{\text {abd }}$ & $6.67 \pm 0.96^{\text {abd }}$ \\
Tanshinone IIA 20- $\mu$ g group & 12 & $15.91 \pm 1.12$ & $7.23 \pm 1.16^{\text {abd }}$ & $9.83 \pm 1.26^{\text {abcd }}$ \\
Tanshinone IIA 40- $\mu$ g group & 12 & $15.79 \pm 1.57$ & $7.45 \pm 2.14^{\text {abd }}$ & $10.29 \pm 2.95^{\text {abcd }}$ \\
\hline
\end{tabular}

${ }^{a}$ Compared with the Control group, $\mathrm{P}<0.05 ;{ }^{\mathrm{b}}$ compared with DMSO + Sham group, $\mathrm{P}<0.05 ;{ }^{\mathrm{c}}$ compared with $\mathrm{DMSO}+$ Tumor group, $\mathrm{P}<0.05 ;{ }^{\mathrm{d}}$ compared with Tanshinone IIA + Sham group, $\mathrm{P}<0.05$.

\section{Comparison of IL-1 $\beta$, IL-6, and TNF- $\alpha$ mRNA expression levels in the spinal cord of all animals}

Three weeks post-injection, IL-1 $\beta$, IL-6, and TNF- $\alpha$ mRNA expression levels in the Tanshinone IIA + Sham and DMSO + Sham groups were not statistically different $(\mathrm{P}>0.05)$ from the Control group. The expression levels of IL-1 $\beta$, IL-6, and TNF- $\alpha$ mRNA in the DMSO + Tumor group were significantly higher than in the Control group $(\mathrm{P}<0.05)$, while that in the Tanshinone IIA 20- $\mu \mathrm{g}$ and 40- $\mu \mathrm{g}$ groups were lower than in the DMSO + Tumor group but higher than the Control group ( $\mathrm{P}<0.05$ ). IL-1 $\beta$, IL-6, and TNF- $\alpha$ mRNA expression levels in the Tanshinone IIA $10-\mu \mathrm{g}$ group were not statistically different from the DMSO + Tumor group $(\mathrm{P}>0.05)($ Table 2$)$.

Table 2. Comparison of IL-1 $\beta$, IL- 6 , and TNF- $\alpha$ mRNA expression levels in the spinal cord of mice fromall groups.

\begin{tabular}{llccc}
\hline Groups & N & IL-1 & IL-6 & TNF- $\alpha$ \\
\hline Control group & 6 & 1 & 1 & 1 \\
DMSO + Sham group & 6 & $1.12 \pm 0.21$ & $1.16 \pm 0.13$ & $1.01 \pm 0.18$ \\
DMSO + Tumor group & 6 & $4.91 \pm 1.33^{\mathrm{ab}}$ & $4.54 \pm 1.72^{\mathrm{ab}}$ & $3.95 \pm 1.14^{\mathrm{ab}}$ \\
Tanshinone IIA + Sham group & 6 & $1.02 \pm 0.16^{\mathrm{c}}$ & $1.11 \pm 0.14^{\mathrm{c}}$ & $1.09 \pm 0.17^{\mathrm{c}}$ \\
Tanshinone IIA 10- $\mu$ g group & 6 & $4.32 \pm 1.26^{\mathrm{abd}}$ & $2.79 \pm 0.89^{\mathrm{abbd}}$ & $4.01 \pm 1.18^{\mathrm{abd}}$ \\
Tanshinone IIA 20- $\mu$ g group & 6 & $2.91 \pm 0.91^{\mathrm{abcd}}$ & $2.67 \pm 0.46^{\mathrm{abcd}}$ & $1.93 \pm 0.71^{\mathrm{abcd}}$ \\
Tanshinone IIA 40- $\mu$ g group & 6 & $2.75 \pm 0.81^{\mathrm{abcd}}$ & $1.86 \pm 0.53^{\mathrm{abcd}}$ \\
\hline
\end{tabular}

${ }^{\mathrm{a}}$ Compared with the Control group, $\mathrm{P}<0.05$; ${ }^{\mathrm{b}}$ compared with DMSO + Sham group, $\mathrm{P}<0.05$; ${ }^{\mathrm{c}}$ compared with $\mathrm{DMSO}+$ Tumor group, $\mathrm{P}<0.05 ;{ }^{\mathrm{d}}$ compared with Tanshinone IIA + Sham group, $\mathrm{P}<0.05$. 


\section{DISCUSSION}

This study established a model of bone cancer pain by inoculating NCTC 2472 fibrosarcoma cells into the femoral bone marrow cavity of mice. The behavioral test showed that mice with bone cancer pain presented with progressive hyperpathia, and radiographs showed that the cancer cells invaded and disrupted the bone cortex, indicating that the model was successful. According to traditional Chinese medicine, the pathogenesis of qi stagnation and blood stasis - or Qi and blood weakness - play an important role in the development and progression of cancer pain. Therefore, nourishing and activating the blood, and blood stasisremoval therapy are often used as treatment. S. miltiorrhizae is a herbaceous perennial, used as a type of traditional Chinese medicine. Its root medicinal, as it promotes blood circulation to eliminate blood stasis, soothes the nerves, aids in apocenosis, and relieves pain (Dictionary of Traditional Chinese Medicine, 1986). Research has shown that S. miltiorrhizae may have an analgesic effect; for example, it can relieve the pain associated with herpes zoster (Zhang, 2006) However, the effective ingredients and mechanism of S. miltiorrhizae in analgesia are not known. Its effective constituents are generally divided into two classes: water soluble and liposoluble. Tanshinone IIA belongs to the liposoluble class. It is reported to relieve the neuropathic pain of diabetic rats (Pan et al., 2006). Due to its liposolubility, it can easily penetrate the nervous system. Therefore, Tanshinone IIA is the most likely analgesic constituents in $S$. miltiorrhizae. Research has also shown that Tanshinone IIA can inhibit TNF- $\alpha$, thus limiting its inflammatory effects (Fan et al., 2009). After several intrathecal doses (once a day for 7 days), Tanshinone IIA has shown to dose-dependently restrain the thermal hyperalgesia of mice with bone cancer pain; the drug doses in this experiment were obtained through preliminary experiments. Further research has shown that Tanshinone IIA can reduce the thermal hyperalgesia of mice with bone cancer pain as well as decrease the expression levels of IL-1 $\beta$, IL-6, and TNF- $\alpha$ mRNA. Therefore, one mechanism for Tanshinone IIA action is attenuation of spinal cord inflammation in mice with bone cancer pain. The site of this action may be in the central nervous system. However, further studies are required to establish the specific mechanism involved in the action of Tanshinone IIA on inflammatory factors in order to determine if it has a regulatory effect on other pain signaling pathways.

\section{ACKNOWLEDGMENTS}

Research supported by the Young National Natural Science Fund (\#81300949) and the Jiangsu Youth Science Funds (\#BK2012102).

\section{REFERENCES}

Dictionary of Traditional Chinese Medicine (1986). Shanghai Scientific \& Technical Publishers, 1st edn. Springer press. Fan GW, Gao XM, Wang H, Zhu Y, et al. (2009). The anti-inflammatory activities of Tanshinone IIA, an active component of TCM, are mediated by estrogen receptor activation and inhibition of iNOS. J. Steroid Biochem. Mol Biol. 113: 275-280.

Lin J, Xu LZ, Li Y and Yang SL (2002). Comparison of the contents of tanshinone A in salvia from different areas. China J. Chin. Materia Medica. 27: 153-154.

Lu Y, Zhu L and Gao YJ (2011). Pain-related aversion induces astrocytic reaction and proinflammatory cytokine expression in the anterior cingulate cortex in rats. Brain Res. Bull. 84: 178-182.

Marchand F, Perretti M and McMahon SB (2005). Roles of the immune system in chronic pain. Nat. Rev. Neurosci. 6: $521-532$. 
Pan XL, Zhou QS and Du DP (2006). The effects of tanshinone A on neuropathic pain in diabetic rats. Chin. J. Anesthesiol. 26: 692-695.

Ren BX, Gu XP, Zhu W, Zheng YG, et al. (2011). Intrathecal injection of metabolic glutamic acid receptor subtype 5 antagonists of bone cancer pain in mice pain behavior and the spinal cord level astrocyte activation. The influence of the behavioral medicine and brain tuition. Chin. J. Behav. Med. Sci. 20: 295-297.

Zhang LH. (2006). The efficacy of salvia infusion on analgesic of shingles. Chin. J. Clin. Med. Pract. 6: 190. 